\title{
Phylogenomic analysis of natural selection pressure in Streptococcus genomes
}

\author{
Maria Anisimova*1, Joseph Bielawski2,3, Katherine Dunn² and Ziheng Yang ${ }^{1}$
}

Address: ${ }^{1}$ Department of Biology, University College London, London, UK, ${ }^{2}$ Department of Biology, Dalhousie University, Halifax, Nova Scotia, Canada and ${ }^{3}$ Department of Mathematics and Statistics, Dalhousie University, Halifax, Nova Scotia, Canada

Email: Maria Anisimova* - manisimova@hotmail.com; Joseph Bielawski - j.bielawski@dal.ca; Katherine Dunn - kathy.dunn@dal.ca; Ziheng Yang - z.yang@ucl.ac.uk

* Corresponding author

Published: 30 August 2007

BMC Evolutionary Biology 2007, 7:154 doi:10.1 186/147/-2/48-7-154
Received: 8 March 2007

Accepted: 30 August 2007

This article is available from: http://www.biomedcentral.com/I47|-2/48/7//54

(c) 2007 Anisimova et al; licensee BioMed Central Ltd.

This is an Open Access article distributed under the terms of the Creative Commons Attribution License (http://creativecommons.org/licenses/by/2.0), which permits unrestricted use, distribution, and reproduction in any medium, provided the original work is properly cited.

\begin{abstract}
Background: In comparative analyses of bacterial pathogens, it has been common practice to discriminate between two types of genes: (i) those shared by pathogens and their non-pathogenic relatives (core genes), and (ii) those found exclusively in pathogens (pathogen-specific accessory genes). Rather than attempting to a priori delineate genes into sets more or less relevant to pathogenicity, we took a broad approach to the analysis of Streptococcus species by investigating the strength of natural selection in all clusters of homologous genes. The genus Streptococcus is comprised of a wide variety of both pathogenic and commensal lineages, and we relate our findings to the pre-existing knowledge of Streptococcus virulence factors.
\end{abstract}

Results: Our analysis of 1730 gene clusters revealed 136 cases of positive Darwinian selection, which we suggest is most likely to result from an antagonistic interaction between the host and pathogen at the molecular level. A two-step validation procedure suggests that positive selection was robustly identified in our genomic survey. We found no evidence to support the notion that pathogen specific accessory genes are more likely to be subject to positive selection than core genes. Indeed, we even uncovered a few cases of essential gene evolution by positive selection. Among the gene clusters subject to positive selection, a large fraction $(29 \%$ can be connected to virulence. The most striking finding was that a considerable fraction of the positively selected genes are also known to have tissue specific patterns of expression during invasive disease. As current expression data is far from comprehensive, we suggest that this fraction was underestimated.

Conclusion: Our findings suggest that pathogen specific genes, although a popular focus of research, do not provide a complete picture of the evolutionary dynamics of virulence. The results of this study, and others, support the notion that the products of both core and accessory genes participate in complex networks that comprise the molecular basis of virulence. Future work should seek to understand the evolutionary dynamics of both core and accessory genes as a function of the networks in which they participate.

\section{Background}

The large number of complete bacterial genomes in the public domain (> 390) has opened the way for genomescale studies of pathogenesis, diversity, evolution and 
gene function. There has been particular interest in comparative analyses of bacterial pathogens, and especially studying molecular mechanisms underlying bacterial adaptation, as such analyses are expected to contribute to disease prevention and treatment [e.g., [1,2]]. A typical approach is to discriminate between exclusive subsets of genes; e.g., genes shared among pathogens and their nonpathogenic relatives (core genes) and those genes showing presence-absence polymorphisms (accessory genes) [3]. Most studies of pathogenicity have restricted themselves to pathogen-specific subsets of accessory genes [e.g., [1]]. Only recently has the potential role of core genes in the evolution of pathogenicity been considered [e.g., [2]]. However, the outcome of dividing genes into exclusive subsets is sensitive to the particular sample of the genomes [4], and this could bias an investigation of the origin and maintenance of bacterial virulence.

This paper focuses on the evolution of genes and gene families of streptococci. The genus Streptococcus is comprised of a wide variety (about 48 species) of both pathogenic and commensal gram-positive bacteria, which are found to inhabit a wide range of hosts, including humans, horses, pigs and cows [5]. Within the host, streptococci often colonise the mucosal surfaces of the mouth, nares and pharynx, but also inhabit the skin, heart or muscle tissue. Niche or tissue-specific adaptations of streptococci remain poorly understood [6]. This study includes the three most important streptococcal human pathogens: $S$. pyogenes causes pharyngitis, wound and skin infections, scarlet and rheumatic fever, pneumonia, necrotising fasciitis, acute glomerulonephritis, cellulitis, and toxic shock syndrome [7]; S. agalactiae is known mainly for severe infections in newborns, such as sepsis, meningitis, and pneumonia, but also causes pneumonia and infections of bloodstream, skin, and urinary tract in adults [8]; and $S$. pneumoniae is a major source for pneumonia, meningitis, septicaemia, otitis media and sometimes occult bacteremia [9]. Most other streptococci are part of normal human flora [5], and three such lineages (two $S$. thermophilus and one S. mutans) are included in this study. Even within a nominal species, the genomes of individual strains vary in size and gene content (Table 1). Undoubtedly, some gene content variation among the lineages sampled in this study corresponds to important pathogenic differences between individual strains. Rather than attempting to $a$ priori delineate subsets of streptococci genes into sets more or less relevant to pathogenicity, we adopted an approach focused on clusters of homologous genes. Although such clusters will not necessarily contain genes involved in presence-absence polymorphisms among pathogenic and non-pathogenic species, they will allow for a broader investigation of functional divergence among pathogenic and non-pathogenic lineages of streptococci.
We estimated rates of evolution for gene clusters comprised of known and putative coding regions, categorised them according to their evolutionary patterns, and identified genes and gene families evolving under positive selection. We found that both core and accessory genes are subject to evolution by positive selection, most likely in the form of diversifying selection resulting from the antagonistic interaction between host and parasite. A large fraction of the genes identified as subject to positive selection have functions that can be connected to virulence. Furthermore, $19 \%$ of the positively selected streptococci gene clusters encode proteins that are known to have body-site specific expression pattern during invasive disease; a result which we argue is likely a substantial underestimate. Taken together, the results of this study and others support the notion that the products of both core and auxiliary genes participate in complex networks that comprise the molecular basis of virulence, and that evolution of the participants of such networks contribute significantly to the dynamics of this phenotype over time.

\section{Results and Discussion}

\section{Characteristics of streptococci gene clusters}

We partitioned the twelve complete genomes from five Streptococcus species (Table 1) into 1730 clusters of homologous gene sequences based on the requirement that a cluster must be comprised of more than three distinct sequences and meet a sequence similarity threshold of $50 \%$. When the threshold was lowered to $30 \%, 1822$ homologous gene groups were identified, with 78 of them obtained by a successive clustering procedure (see methods). Both sets of clusters were subjected to identical data analysis procedures. Despite the clustering differences, the results for both sets were consistent: the same conclusions were drawn from the estimates of evolutionary parameters, and the sets of genes detected to be under positive selection were very similar. This indicates a level of robustness of our results to the details of the clustering procedure. We therefore show results using the set of homologous gene clusters based on the 50\% similarity threshold (unless stated otherwise). In this case, the size of clusters varied from 3 to 59 sequences, and almost a third of clusters (574) contained at least 12 sequences. Sequence length ranged from 300 (the lower bound on our selection criterion) to 4944 nucleotides, the average being 1027 nucleotides.

As expected, the sampled streptococci genomes were ATrich, with nucleotide content varying among different codon positions, and among genes and genomes. The average AT content of the sampled genomes ranged from 60 to $64 \%$, and the average for the gene clusters ranged from $49-74 \%$. AT content at $2^{\text {nd }}$ and $3^{\text {rd }}$ codon positions (65\% and 68\% respectively) was more biased as compared to $1^{\text {st }}$ codon positions (49\%). For certain genes AT 
Table I: Twelve complete genomes of congeneric Streptococcus used in this study

\begin{tabular}{|c|c|c|c|c|}
\hline Streptococcus strain & Genbank accession no. & Genome size (bp) & $\begin{array}{l}\text { No. of CDs (>99 } \\
\text { codons) }\end{array}$ & Reference \\
\hline S. pyogenes MI GAS & NC 002737 & $|, 852,44|$ & 1,697 (1509) & {$[60]$} \\
\hline S. pyogenes MGAS8232 & NC 003485 & $1,895,017$ & I,845 (I584) & [7] \\
\hline S. pyogenes MGAS3I5 & NC 004070 & $1,900,521$ & I,865 (1596) & [6I] \\
\hline S. pyogenes SSI-I & $\overline{N C 004606}$ & $\mathrm{I}, 894,275$ & I,86I (I573) & {$[62]$} \\
\hline S. pyogenes MGASI 0394 & NC 006086 & I,899,877 & $1,886(1582)$ & [63] \\
\hline S. agalactiae NEM3I 6 & NC 004368 & $2,211,485$ & $2,094(1887)$ & [8] \\
\hline S. agalactiae $2603 \mathrm{~V} / \mathrm{R}$ & NC 004116 & $2,160,267$ & $2,124(1831)$ & {$[64]$} \\
\hline S. pneumoniae R6 & NC 003098 & $2,038,615$ & $2,043(1723)$ & {$[65]$} \\
\hline S. pneumoniae TIGR4 & NC 003028 & $2,160,837$ & $2,094(|70|)$ & [66] \\
\hline S. mutans UAI59 & NC 004350 & $2,030,921$ & $1,960(1690)$ & [67] \\
\hline S. thermophilus LMG I83II & $\overline{N C \quad 006448}$ & $1,796,846$ & $\mathrm{I}, 889(154 \mathrm{I})$ & [68] \\
\hline S. thermophilus CNRZI066 & NC 006449 & $1,796,226$ & $1,915(1554)$ & {$[68]$} \\
\hline
\end{tabular}

content was extremely high at $3^{\text {rd }}$ codon positions while overall AT content was comparatively low: the majority of these cases were $30 \mathrm{~S}$ and $50 \mathrm{~S}$ ribosomal proteins ( $r p s A, E$, $I, K, M$ and $r p l B, D, J, K, L, O, Q)$ as well as uridine phosphorylase $(u d p)$, protein GRAB (known virulence factor), cell division protein (ftsZ), alcohol dehydrogenase I (adhA, $P, 1)$, cysteine synthase, O-acetylserine sulfhydrylase (cys $M, M 1, K)$, triose phosphate isomerase (tpi), cellwall surface anchor family protein and a homologous cluster containing glucan binding protein ( $p c s B)$, secreted antigen $(g b p B / s a g A)$, and general stress protein GSP-781.

Transition/transversion $(\kappa)$ ratios varied widely among the gene clusters: $95 \%$ of gene clusters had $\kappa$ between 1 and 20 , with the average ratio being 2 . At the tails of the distribution we also found $2.8 \%$ of gene clusters with no transversions (forcing the estimate of $\kappa$ to the upper boundary) and $2 \%$ of gene clusters with $\kappa<1$. The latter cases included B-cell receptor associated protein, minor capsid protein, peptidoglycan hydrolase (mur1), ABC transporter membrane-spanning permease $(f a t D$; iron transport), bacterocin transport accessory (bta), oxidoreductase $(\operatorname{moc} A)$, acetylglutamate kinase $(\arg B)$, and pyrroline carboxylate reductase (proC).

We used the cluster-specific gene trees, as estimated by PHYML [10], as the basis to estimate the mean number of changes per codon per branch under model $\mathrm{M} 0$, as implemented in PAML [11]. In 95\% of the clusters the mean amount of codon evolution was between 0.003 and 8 substitutions per codon site. However, in a small fraction of clusters $(0.5 \%)$ we observed either a very low amount of codon evolution $(<0.001$ for orthologues from the same species, $S$. pyogenes), or a very high amount of codon evolution ( $>20$ due to excess of synonymous substitutions in orthologues from different species).
Extreme outliers for AT content, transition/transversion ratio, and high amount of codon evolution are strong candidates for genes having a history of lateral gene transfer between members of the genus Streptococcus and more distantly related outgroup lineages [e.g., $[12,13]]$. We note however, that a large amount of codon evolution might also arise as a consequence of strong positive Darwinian selection.

\section{Phylogenomic analysis of evolutionary relationships among lineages}

A subset of the gene clusters were concatenated for the purpose of obtaining a genome-scale estimate of the Streptococcus phylogeny. We restricted our phylogenetic analysis to the 504 clusters that were comprised of exactly one gene from each of the 12 genomes, as identified under the $30 \%$ threshold. Gene sequences from those 504 clusters were concatenated (555,297 nucleotides), and a phylogenetic analysis of this dataset resulted in the phylogenetic tree shown in Figure 1. This genome-scale estimate of relationships among species agrees with some prior estimates based on smaller molecular datasets $[14,15]$. The position of the root can be deduced by using the outgroup species Lactococcus lactis. Examination of the phylogenies estimated from each of the 504 single-gene clusters revealed that only $26 \%$ matched the species level relationship estimated from the concatenated data. Given such a high level of among gene variability, and the possibility that some portion could reflect inter-species recombination events [e.g., [16]], all subsequent analyses were based on cluster-specific estimates of the gene tree.

The length of the tree estimated from the concatenated data was 1.75 nucleotide substitutions per site. Tree length taken as an average over all the single-copy gene clusters was biased by the presence of trees with long lengths, where estimation problems were likely. After excluding $5 \%$ of trees having the largest estimated length, 


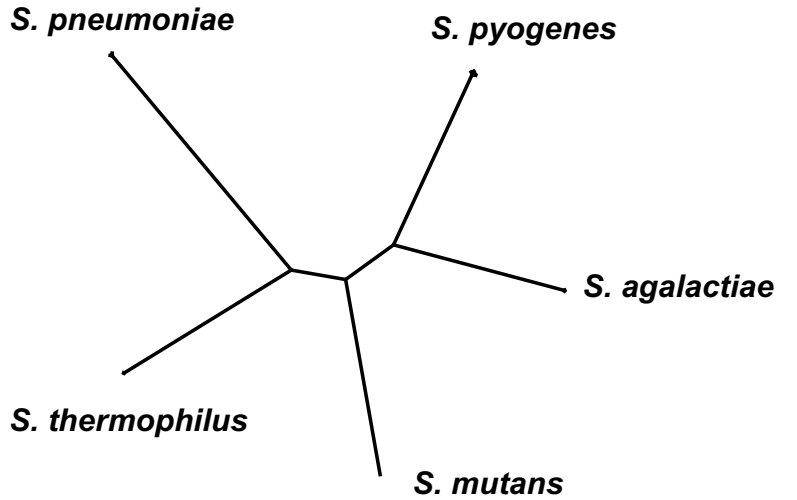

1.0

\section{Figure I}

A genome-scale estimate of phylogenetic relationships among species of Streptococcus. The tree topology is derived from the joint analysis of 504 single-copy gene sequences by using the BIONJ algorithm [55]. Each gene was comprised of exactly one sequence from each of the 12 genomes; the topology shown above is simplified to show only the relationships among the five nominal species of Streptococcus. The topology is unrooted, and branch lengths indicate the mean number of substitutions per codon, as inferred under codon model M0 [I I].

the average tree length was 3.53 nucleotide substitutions per site, with 95\% between 1.4 and 51.2. According to simulations [17] high accuracy of ML estimation, and the LRTs, is expected under such levels of sequence divergence. The shortest trees ( 0.8 to 1.5$)$ were obtained from 30S, 50S ribosomal proteins $(r p s B, C, D, G, K, L, M$ and rplC, $E, N, P, Q, T, V)$ and translation elongation factors (tufA, fusA). The longest trees $(\geq 50)$ were obtained from membrane-spanning proteins, cell division proteins (divlC, ftsL), ATP-NAD kinase ( $p p n K)$, pantothenate kinase (coaA), diacylglycerol kinase ( $d g k, d g k A, d a g K)$, exfoliative toxin $A$, exotoxin $B$ (eetB, shet $A)$, 3-dehydroquinate dehydratase (aroD), enoyl CoA hydratase II ( $p h a B)$, and isopentenyl diphosphate isomerase (idi, fni). For genes of such high divergences the LRT has almost no power to detect positive selection, although the Bayesian prediction of sites under positive selection is expected to be highly unreliable in such cases $[17,18]$.

\section{A two-step "genome scan" indicates robust identification of positive selection}

Gene clusters identified as subject to positive selection pressure are listed in Additional file 1. We considered the signal of positive selection to be strong when both LRTs (M1a versus M2a and M7 versus M8) were significant at
5\%; 38 gene clusters met this criterion (see Additional file 1). An additional 98 gene clusters were identified by the LRT of models M7 and M8 at the 5\% significance level (see Additional file 1). For the latter cases, it is possible that model $\mathrm{M} 2 \mathrm{a}$ was too conservative to detect positive selection. Indeed, simulation studies indicate that the LRT of M1a versus $\mathrm{M} 2 \mathrm{a}$ is less powerful than the LRT of M7 versus M8 in such cases [17].

Any genome-scale study that surveys for positive selection is susceptible to false positives arising from uncertainty in parameter estimation [17], a history of recombination $[19,20]$ or from procedural artefacts such as alignment errors. However, such surveys provide a powerful means to generate novel biological hypotheses for further experimental and statistical analysis [e.g., [2]]. To assess the potential impact of such errors on our investigation, we performed a substantially expanded analysis on a sample of genes we detected to be evolving under positive selection. To minimise the effect of uncertainty in parameter estimation, we compiled a larger set of gene sequences. To avoid potential for procedural artefacts, we analysed the data "by hand" rather than using our tools to automate the process; this included careful visual inspection and editing of the sequence alignments. Lastly, we employed the methods of Wilson and McVean [21] to evaluate the possibility of positive selection while simultaneously allowing for a history of recombination.

Clusters of genes initially identified as subject to positive selection were pre-screened for those comprised of a small number of sequences. Eight genes were then randomly chosen from this subset, conditional on the availability of additional homologous sequences in GenBank. We chose the ninth gene, $r p l S$, because positive selection was unexpected, and the tenth gene, IS1191 transposase, because it is found only in $S$. thermophilus. All ten datasets were expanded by sampling homologous sequences from additional lineages of streptococci. We note that the distribution of strains among the streptococcal species was comparable. Genes subjected to expanded data analyses are listed in Table 2. Reanalysis under the standard set of models revealed that all ten datasets retained a signal of positive selection, six of which were significant at $1 \%$, and two were significant at 5\%, under both of the LRTs (Table 2 ). The remaining two datasets, rplS and a hypothetical protein-coding gene homologous to sib38, were significant only under the LRT comparing M7 and M8. We next employed the approach of Wilson and McVean [21] to evaluate the possibility that both selection and recombination might have impacted the evolution of these data. In each case the results of this approach, which does not suffer from high false positive rates stemming from a history of recombination, confirmed positive Darwinian selection (data not shown). 
Table 2: Expanded analyses of ten genes detected to be under positive selection in a genome scan

\begin{tabular}{|c|c|c|c|c|c|c|c|c|}
\hline \multirow[b]{2}{*}{ Gene } & \multirow[b]{2}{*}{ Species } & \multirow[b]{2}{*}{$\mathbf{N}_{\mathbf{l}}$} & \multirow[b]{2}{*}{$\mathbf{N}_{2}$} & \multirow[b]{2}{*}{$L_{n t}$} & \multicolumn{2}{|c|}{$P$-value of LRT } & \multirow[b]{2}{*}{$p_{1}$ in $M 8$} & \multirow[b]{2}{*}{$\omega$ in $\mathbf{M 8}$} \\
\hline & & & & & Mla vs M2a & M7 vs $M 8$ & & \\
\hline $\begin{array}{l}\text { Cell envelope proteinase } \\
\text { (prtS); }\end{array}$ & S. pyogenes & 5 & 28 & 4947 & 0.0000 & 0.0000 & 0.010 & 15.0 \\
\hline \multirow{3}{*}{$\begin{array}{l}\text { Lactocepin Dipeptidase } \\
\text { (pepD) }\end{array}$} & S. pyogenes & 8 & 20 & 1416 & 0.0002 & 0.0000 & 0.016 & 8.0 \\
\hline & S. agalactiae & & & & & & & \\
\hline & S. mutans & & & & & & & \\
\hline $\begin{array}{l}\text { Ftsk/Spolll family } \\
\text { protein }^{\text {a }}\end{array}$ & S. pyogenes & 4 & 10 & 756 & 0.0000 & 0.0000 & 0.049 & $\infty$ \\
\hline Hypothetical protein & S. pyogenes & 4 & 10 & 1098 & 0.0000 & 0.0000 & 0.017 & 27.5 \\
\hline $\begin{array}{l}\text { ISII9I transposase } \\
\text { (truncated) }\end{array}$ & S. thermophilus & 4 & 6 & 372 & 0.0020 & 0.0020 & 0.087 & 25.0 \\
\hline Hypothetical protein & S. pyogenes & 3 & 13 & 1092 & 0.0491 & 0.0004 & 0.033 & 12.4 \\
\hline Hypothetical proteinc & S. pyogenes & 4 & 7 & 402 & 0.0214 & 0.0214 & 0.026 & 31.2 \\
\hline grab & S. pyogenes & 3 & 11 & 735 & 0.0035 & 0.0034 & 0.062 & 15.5 \\
\hline rplSd & all & II & 30 & 345 & 0.1254 & 0.0080 & 0.010 & 6.7 \\
\hline Hypothetical proteine & S. pyogenes & 4 & 13 & 1023 & 0.0679 & 0.0442 & 0.18 & 2.6 \\
\hline $\begin{array}{l}\text { Homologue of macl } \\
\text { sib38 }\end{array}$ & & & & & & & & \\
\hline
\end{tabular}

Notes: $N_{1}$ indicates the number of gene sequences in the original gene cluster. $N_{2}$ indicates the number of sequences in the expanded dataset. $L_{n t}$ is the length of the gene sequence in nucleotides. $p_{1}$ indicates the proportion of codon sites estimate to be subject to positive selection under model M8.

$\omega$ is the parameter in the codon model M8 for the nonsyonymous to synonymous rate ratio $\left(d_{N} / d_{S}\right)$.

Additional remarks:

a Best non-GAS blastp hit: gb|AAK79835.I|AE007695 8 (AE007695) FtsK-like DNA segregation ATPase, YDCQ B. subtilis orthologue [Clostridium acetobutylicum];

b Fibronectin-binding protein (probable antigen);

c Best non-GAS blastp hit: gb|AAB97959.1] (U96166) ATP-binding cassette lipoprotein [S. cristatus];

d May play a role in the structure and function of the aminoacyl-tRNA binding site;

e Novel immunoglobulin binding protein; best blastp hit: sp|PI I 2 I5|ITAM_human cell surface glycoprotein MAC-I $\alpha$ subunit precursor (CR-3 $\alpha$

chain) (CDIIB) (leukocyte adhesion receptor MOI) (integrin $\alpha-M)$ (neutrophil adherence receptor).

The two cases where the LRT comparing models M1a and M2a failed to detect a signal of positive selection appears to reflect the greater restrictions on the parameter space of M1a and M2a as compared with M7 and M8. M1a and M2a explicitly model a class of purely neutral sites $(\omega=1)$ although it might not exist in a given data set; in which case sites having $\omega=1$ could reflect an artefact of averaging over sites subject to moderate positive selection and other sites subject to purifying selection. Indeed, when we examined the parameter estimates under M8 for these two genes we observed (i) a very large fraction of sites $(80 \%-$ $82 \%)$ were subject to very strong purifying selection $(\omega \approx$ 0.006 ) or moderate purifying selection ( $\omega$ ranging from 0.11 to 0.36 ) and (ii) the absence of a purely neutral site class. These empirical results are consistent with the results of a simulation study [17] which showed that the LRT of M1 and M2 are often less powerful than the LRT of $\mathrm{M} 7$ and M8 in similar cases.

In general, by expanding these 10 datasets we increased the numbers of lineages and levels of sequence divergence (Table 2) to values where the LRTs are expected to have low type I error rates [17]. This result, taken together with robust detection of positive selection (Table 2), suggests that positive section was reliably identified from our ten samples of streptococci gene sequences. We note that large scale surveys for recombination using traditional detection software [e.g., [22]] are possible, but such programs likely suffer from an inverse effect, i.e. recombination may be falsely detected due to the presence of positive selection. Unfortunately, it is computationally prohibitive to carry out the approach of Wilson and McVean [21] for all genes in a genome. For the time being, we suggest the two-step process carried out in this study will provide a valuable means to gauge the robustness of selected cases obtained from such "genomic scans".

\section{A large fraction of genes subject to positive selection are connected to virulence}

Since both orthologous and paralogous genes can be included in the same gene cluster, positive selection detected in a cluster can be a result of various processes: adaptation of a species to optimise the process of infection, escape host immune response, adaptation to a different environmental niches (e.g., milk in S. thermophilus, or dental flora in S. mutans), and functional diversification 
of members of multi-gene families. Indeed, the 136 clusters identified as having evolved under positive selection were connected to a wide variety of functions (see Additional file 1 ), with only $10 \%$ having no ascribable function. Interestingly, $7 \%$ of the clusters were comprised of genes known to be essential to $S$. pneumoniae (see Additional file 1), i.e. those where experimental disruption was shown to be lethal [23], indicating that even essential genes can be subject to rapid evolution by natural selection. A substantial fraction of the clusters, (29\%) had either a known, or hypothesised, connection to virulence in streptococci (see Additional file 1). We considered a gene to have a connection to virulence if it was related to survival, spread, or persistence within its human host $[24,25]$. In the remainder of this section we focus on a select subset of the virulence genes subject to positive selection.

Three genes, grab, endoS, and ideS, are well known to play a critical role in the ability of pathogenic streptococci to evade detection by the host immune system. The grab gene encodes the G-related $\alpha_{2}$ macroglobulin-binding protein (GRAB) which functions to inhibit host-mediated proteolysis, a defence process that is often activated by the host during invasive infection [26].

GRAB mediates the binding of human $\alpha-2 M$ (the dominant proteinase inhibitor of human plasma) to the surface of $S$. pyogenes thereby conferring protection against proteolytic degradation of important virulence determinants $[26,27]$. EndoS is an extracellular protein, encoded by the endoS gene, which hydrolyzes N-linked oligosaccharides on the heavy chains of immunoglobulin G (IgG), thereby impacting IgG function and preventing opsinising antibodies from triggering a protective immune response to $S$ pyogenes [28]. The highly specific activity of this endoglycosidase increases pathogen survival in human blood containing opsonising antibodies [28-30]. Finally Ides (or streptococcal Mac-1) is a secreted cysteine proteinase that cleaves the heavy chain of IgG and thereby also inhibits the susceptibility of $S$. pyogenes to phagocytosis [31]. The IdeS protein is specific to IgG antibodies and has been found to be expressed in clinically important serotypes. In each case above, the function of the protein is to modulate a critical host defence molecule. This creates a situation where an evolutionary arms-race between pathogen and host is likely, with the host subject to intense selection pressures to avoid such modulation of its defence systems by the pathogen. Our finding that each of these genes has evolved under positive selection pressure is consistent with such an antagonistic interaction between host and pathogen at the molecular level, and indicates that such evolutionary interactions are characteristic of several different systems used by pathogenic streptococci to escape recognition by the host. In each of the above systems the positive fitness consequences to the pathogen to winning such an arms race appears to be increased survival in human blood $[28,31]$.

Escape from immune recognition is just one aspect of the ability of a pathogen to successfully invade and colonise its host. By conducting a genome-scale analysis of positive selection we identified sets of genes, when taken together with data derived from alternative methods of investigating virulence factors, provided insights into other potentially important molecular systems connected to streptococci virulence. For example, heat shock genes have been connected to bacterial virulence [32-34]. The products of heat shock genes are typically thought of as contributing to a protective cellular response to cope with the stress-induced damage of proteins. As such, many heat shock proteins are molecular chaperones or ATP-dependent proteases that play important roles in protein folding, repair, and degradation [34-36]. However, Charpentier and coworkers [37] also reported that ClpC proteases plays a major role in processes related to streptococci virulence, including adherence to human cells and production of known virulence factors such as pneumolysin, autolysin $\mathrm{A}, \mathrm{CbpA}$, and other choline binding proteins. Recently, Ibrahim et al. [34] suggested ClpC may play a major role in the virulence of $S$. pneumoniae. Consistent with a potentially important role in virulence, we found ATP-dependent proteases encoded by the $c l p L, c l p C$ genes have evolved under positive selection pressure. We note that a signature-tagged mutagenesis screen of $S$. pneumoniae also identified ClpC as a virulence factor [38], and that $\mathrm{ClpC}$ has been shown to contribute to the ability of $S$. pneumoniae to grow in the lungs and blood [34]. Interestingly, we identified several other genes known to have a role in responding to stress conditions ( $r e l A, g l s 24$ and $a t p D(a t p B)$ as having evolved under positive selection.

Efficient genome replication is essential for growth and survival of an organism, and polymerase complexes often fail to complete this task $[39,40]$. For some pathogenic bacteria this is especially important, as successful replication is thought to contribute to proliferation and efficiency of the colonisation of hostile environments [e.g., [41,42]]. Interestingly, we detected positive selection in several single-copy protein-coding genes connected to replication, recombination and repair proteins (singlecopy genes $r e c G, r e c N, r e c R, r e c M$ ), and in two ribosomal proteins (rpsB and rplS) and two DNA-dependent RNA polymerases (rpoC and rpoB). Replication forks often stall and may collapse to generate new DNA ends that provoke recombination and induce genomic rearrangements $[40,43]$. Complete replication of the genome depends therefore on repair activities to remove or bypass lesions in the DNA, modulation of RNA polymerase to reduce conflicts with transcription, and recombination systems 
to rescue forks that have stalled or collapsed. In these cases the nature of selection pressure is far from clear. Selection pressure on these genes could reflect constraints on efficient genome replication during colonisation and proliferation in the hostile environment of the host [e.g., $[41,42]]$, or perhaps the ability to recombine during mixed colonisation by unrelated strains [e.g., [44]]. Neither is it known if these genes might be involved in an antagonistic interaction between host and pathogen at the molecular level.

Generally speaking, our genome-scale analysis of streptococci protein-coding genes uncovered a wide array of clusters that can be connected to virulence and which are subject to positive selection pressure. In some cases the nature of the selection pressure seems clear, e.g., grab, endos, and ides described above. We documented other cases where we identified genes previously hypothesised to play an important role in virulence but where current data were not conclusive. Yet another example of this is the two-component signal transduction systems mediated by histidine kinase; they are integral parts of bacterial cellular regulatory processes, and are used to regulate the expression of genes involved in virulence [25,45]. We identified five clusters comprised of genes from two-component response regulation systems, with two cases (ihk and ciaR) having been previously hypothesised to play an important role in virulence $[34,46,47]$. We believe that large-scale analyses such as ours play an important role in highlighting particular molecular systems that warrant further study. Finally, there were some cases where the nature of selection pressure was unclear, e.g., replication, recombination and repair proteins $r e c G, r e c N, \operatorname{rec} R, \operatorname{rec} M$ described above. Identification of such genes helps to generate new hypotheses concerning the role of different molecular systems in virulence.

\section{Both the accessory genome and the core genome are subject to positive Darwinian selection}

A typical approach to studying the evolution of pathogenicity is to focus on the subset of genes found exclusively in the genomes of pathogens [e.g., [1]]. Hereafter we refer to these genes as pathogen-specific accessory genes. Clearly the acquisition of such accessory genes by lateral gene transfer (LGT) plays an important role in the acquisition of characteristics necessary for a pathogenic lifestyle [e.g., [1,3]]. Since pathogens are typically involved in a co-evolutionary arms race with their hosts [48], one might expect pathogen-specific accessory genes are more likely to be subject to diversifying selection. Indeed, a recent study suggested that among recently transferred genes in $S$. pyogenes there is a higher relative frequency of positive selection [15]. To test if this notion is broadly applicable to all pathogen-specific genes we compared clusters of pathogen-specific accessory genes to clusters of core genes (clusters comprised of exactly one of each lineage of Streptococcus). Indeed, there were more pathogen-specific clusters (692) than core-gene clusters (526); however, the ratio of significant to non-significant cases for positive selection did not differ significantly between the pathogen-specific (44/648) and core gene clusters $(41 / 485)$, as indicated by a Fisher's exact test $(P=$ $0.364)$. We also tested pathogen-specific accessory genes against non-pathogen specific accessory genes, and found no evidence for a significant difference $(P=0.761)$. We note that a fraction of these gene clusters are likely have been impacted by gene duplication and deletion events, as well as by LGT.

Marri et al. [15] found that the relative frequency of positive selection was 3 fold higher in recently transferred genes in $S$. pyogenes as compared to core genes. As S. pyogenes causes a wider variety of human diseases than any other bacterial species, and possesses the largest number lineage-specific genes (381), we wanted to investigate the possibility that $S$. pyogenes also has a greater frequency of positive selection among its unique accessory genes. A chisquare test indicated that the odds of positive selection did not differ significantly among clusters of accessory genes unique to a given species of Streptococcus $\left(\chi^{2}=\right.$ $5.122, \mathrm{df}=4, P=0.275)$. To minimise the impact of gene duplication and deletion, and to make a more direct comparison to the study of LGT by Marri et al. [15], we identified the clusters comprised of exactly one of each of the five sampled lineages of $S$. pyogenes and absent for all the other genomes under study. The relative frequency of positive selection was similar in both the single-copy $S$. pyogenes accessory genes (17/207) and in the streptococci core gene clusters $(41 / 526)$, being about $8 \%$. We note that Marri et al. [15] employed the same LRT (M7 against M8) to identify positively selected genes. It appears that the finding of higher odds of positive selection among recently transferred genes [15] could be sensitive to the method of assembly and classification of gene clusters.

As functional category has been shown to be related to selection pressure [49], we investigated the possibility that functional category could have been a confounding factor in our tests for heterogeneity between pathogen-specific gene clusters and core gene clusters. We subdivided clusters among 6 functional categories based on COG assignments and computed the odds of positive selection for pathogen-specific accessory gene clusters and core gene clusters (Table 3). Although the highest odds of positive selection among the pathogen-specific clusters was associated with cellular processes and signalling, the odds ratio for this functional category was 1.04, indicating that a high frequency of positive selection also occurred among the core gene clusters in this category. The highest odds ratio in favour of pathogen-specific accessory genes (2.92) 
did not differ significantly from one (Table 3). Indeed, the only category in which the odds of positive selection differed significantly between pathogen-specific accessory genes and core genes was for metabolism; and, in this case the odds of positive selection were higher for core gene clusters (Table 3 ).

Our findings clearly indicate that the genes involved in presence-absence polymorphism among lineages of streptococci do not provide a complete picture of the nature of pathogen evolution within this genus, as core gene clusters have a similar propensity for positive selection pressure as pathogen-specific gene clusters. The properties of the analytical models used in this study provide some insight into this finding. LRTs based on the site-models we used are best suited to the discovery of genes having rapid amino acid change over long periods of time [e.g., [17]]. Among streptococci, this is most likely to arise in response to the antagonistic evolutionary arms-race between pathogen and host. As such an evolutionary interaction leads to diversifying selection pressure, where fitness advantages are conferred by possessing an allele that is merely different from others in the population; the 136 genes identified as subject to positive selection pressure are the best candidates for such a mode of evolution. Hence, our results reflect that Streptococcus pathogen evolution may be thought of having at least two components: (i) the acquisition by LGT of accessory genes that confer characteristics necessary for a pathogenic lifestyle, and (ii) the long term evolutionary arms race between host and pathogen that impacts the evolution of certain core and accessory genes. We note that other modes of adaptive evolution are plausible, such as a burst of adaptive evolution in an accessory gene following an LGT event or long term divergent selection pressure between pathogenic and non-pathogenic lineages of streptococci, but they are not detectible by using the codon models employed in this study.

\section{A large fraction of positively selected genes have tissue specific patterns of expression}

In vivo gene expression studies of invasive disease have provided insights into the response of bacteria to the host during the course of infection by revealing that known virulence factors, along with other types of genes, display body-site specific expression patterns $[6,50]$. Such knowledge has lead to prediction that several streptococci genes of unknown function contribute to virulence, with these predictions being confirmed by experimentation [6]. However, extensive body-site specific contributions to pathogenesis make it difficult to determine if there is a core set of virulence-related genes required by all streptococci, or how extensive such a set of genes might be. Given such variability, we were interested to determine (i) what fraction of positively selected genes, if any, exhibited body-site specific patterns of gene expression during invasive disease, and (ii) if the intensity of selection might depend on their putative cellular role, as defined by level of gene expression.

We obtained expression data from the large-scale analysis of $S$. pneumoniae by Orihuela et al. [6] which provides data for expression levels within infected blood (IB), cerebrospinal fluid (CSF) and epithelial cell contact (ECC). Taken

Table 3: Odds of positive selection in gene clusters categorised according to COG-derived functional assignments

\begin{tabular}{|c|c|c|c|c|c|}
\hline & \multicolumn{3}{|c|}{ Odds of positive selection } & \multicolumn{2}{|c|}{ Pathogen verses core clusters } \\
\hline & All clusters & $\begin{array}{c}\text { Pathogen-specific } \\
\text { clusters }\end{array}$ & Core-gene clusters & Odds ratio & $P$-value \\
\hline $\begin{array}{l}\text { Information } \\
\text { storage \& } \\
\text { processing }\end{array}$ & 0.07 & 0.05 & 0.08 & 0.61 & 0.565 \\
\hline $\begin{array}{l}\text { Cellular processes } \\
\& \text { signalling }\end{array}$ & 0.10 & 0.10 & 0.10 & 1.04 & 1 \\
\hline Metabolism & 0.08 & 0.04 & 0.12 & 0.35 & 0.02158 \\
\hline $\begin{array}{l}\text { Gene with } \\
\text { inadequately } \\
\text { characterised } \\
\text { function }\end{array}$ & 0.05 & 0.07 & 0.02 & 2.92 & 0.2802 \\
\hline $\begin{array}{l}\text { Not in the COG } \\
\text { database }\end{array}$ & 0.08 & 0.08 & 0.00 & Infinite & 1 \\
\hline $\begin{array}{l}\text { Genes with } \\
\text { multiple COG } \\
\text { functions }\end{array}$ & 0.07 & 0.06 & 0.09 & 0.68 & 1 \\
\hline
\end{tabular}

Notes: The odds of positive selection were computed as the relative frequently of genes under positive selection divided by the relative frequency of genes not under positive selection. The odds ratio is simply the ratio of the odds of positive selection in two different categories of genes. The categories were based on functional assignments found in the COG database. The hypothesis that an odds ratio differed from one was tested by using the Fisher's exact test. 
over all tissue types, 26 (19\%) of the positively selected gene clusters encode proteins that are known to have body-site specific expression. However, the expression data is derived only from $S$. pneumoniae, and patterns of gene expression are thought to vary among pathogens. Interestingly, when we excluded the 47 gene clusters that did not contain at least one representative of $S$. pneumoniae, we found that the relative frequency of genes with body-site specific expression increased to $29 \%$. The results are presented according to tissue-type in Figure 2. These data suggest a disparity among tissue types on the intensity of diversifying selection pressure, in so far as there were more positively selected genes having altered expression in CSF and ECC tissue types than in the other categories (Figure 2). Note that all the genes contributing to Figure 2 have $>2$ fold difference in tissue specific expression levels during invasive disease [6].

Current expression data is far from comprehensive, as it does not cover all body sites, variability among lineages of pathogens, nor even cover all the genes of $S$. pneumoniae. For this reason, we expect that we have underestimated the numbers of positively selected genes that have bodysite specific expression during invasive disease. Large scale studies of expression level, signature tagged mutagenesis, and differential fluorescence induction are beginning to reveal that virulence is a function of a network of genes [e.g., $[6,50,51]]$, many participants being core genes that are not well characterised. Indeed, our finding that core genes, and even essential genes, can be subject to an antagonistic evolutionary arms-race between pathogen and host also highlights the need to gain a better understanding of the role core gene products have in virulence. We look forward to the time when gene networks are better understood, particularly with respect to lineage-specific alterations in the pattern of transcription during invasive disease. An understanding of the evolutionary dynamics of virulence factors in such a context offers the potential to identify previously unappreciated targets for vaccines or pharmaceuticals $[6,51]$.

\section{Conclusion}

Based on our genome-wide analysis of selection pressure, we argue that the evolution of pathogen specific genes, although a popular focus of research, does not provide a complete picture of the evolutionary dynamics of virulence. There is no doubt that the acquisition of novel genes by LGT is critical to the colonisation and exploitation of novel niches, including the pathogenic lifestyle. However, those genes acquired by LGT impinge upon a complex network of interactions among gene products embedded in cellular metabolism. The effect of an LGT could be wide-spread in that the acquired gene or genes could have direct interactions with core gene products or result in an altered cellular environment (perhaps simply

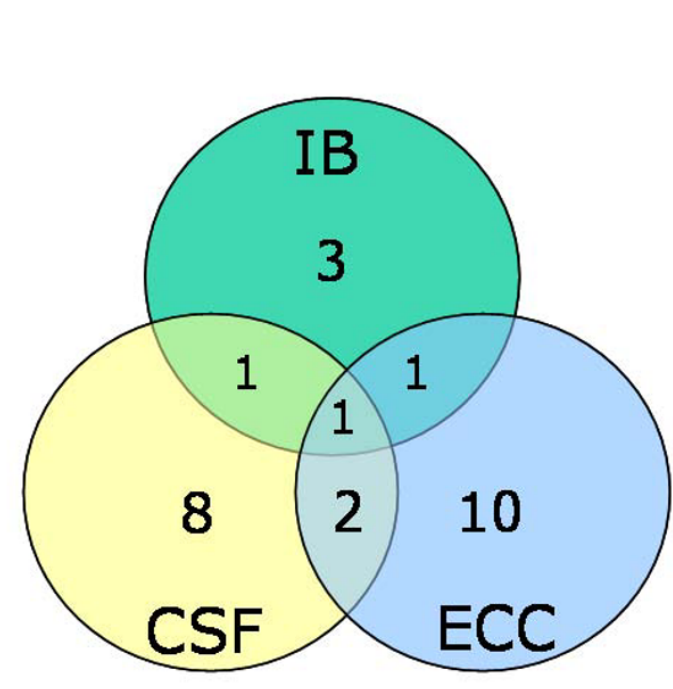

\section{Figure 2}

A Venn diagram showing the distribution of 26 proteins known to have body-site specific patterns of gene expression during invasive disease in Streptococcus pyogenes and to have evolved under positive Darwinian selection. Expression data are from Orihuela et al. [6] for infected blood (IB), cerebrospinal fluid (CSF) and epithelial cell contact (ECC).

by allowing colonisation of a new niche) such that selective pressures acting on core gene products are impacted. As we gain a better understating of how niche specialisation, and pathogenicity in particular, is a function of networks of genes, we can begin to understand the evolutionary dynamics of individual core and auxiliary genes as a function of the networks in which they participate. The findings of this study, and others [e.g., $[6,25]]$, suggest that the origin and evolution of the molecular mechanisms that result in enhanced virulence are more complex than previously thought.

\section{Methods}

Data, homologue clustering, and alignment

Twelve complete genomes of five Streptococcus species (available in Genbank) were analyzed in this paper: five strains of $S$. pyogenes (NC_004070, NC 003485, NC 002737, NC 006086, NC 004606), two strains of $S$. agalactiae ( $\mathrm{NC} \mathrm{004368,}$ NC 004116), two strains of $S$. pneumoniae (NC 003098, NC 003028), one strain of $S$. mutans (NC_004350), and two strains of S. thermophilus (NC 006448, NC 006449). Table 1 summarises the key details of the sampled genomes. All known and putative codon regions longer than 100 codons were extracted from the genomes and the correct reading frame was preserved (i.e., every sequence started with the first codon position). None of the extracted sequences contained in- 
frame stop codons, and stop codons at the end of the sequences were deleted. The amino acid library was created by translating each DNA coding sequence into an amino acid sequence. We separated the amino acid sequences into homologous clusters by using BLASTCLUST program [52], which finds pairs of sequences that have statistically significant matches (using the BLAST algorithm) and groups them using single-linkage clustering. Two distinct sets of homologous clusters were produced based on minimum $30 \%$ and $50 \%$ similarity. Other clustering criteria were: $90 \%$ minimum length coverage and $10^{-6}$ cut-off e-value. As a result, each cluster can contain both orthologous and paralogous sequences. Both sets of clusters were analysed using the procedure described below.

Each cluster of amino acid sequences was aligned using TCoffee [53]. The alignments were based on BLOSUM62 matrix with gap opening and gap extension penalties -5 and -2 respectively. Codon alignments were created from the amino acid alignments by inserting three-nucleotide gaps into corresponding DNA sequences. Only clusters with at least three sequences were used for further analyses. Summary statistics were collected using PAUP* 4.0 [54], and ML trees were reconstructed from DNA data using a heuristic search implemented in PHYML [10].

\section{Assessment of alignments}

The set of clusters based on $30 \%$ similarity threshold were more divergent; consequently, for some large clusters alignments were problematic. In contrast, even the largest clusters based on the $50 \%$ similarity had acceptable alignments. In the absence of an objective universal score to compare alignments of different datasets, we chose the 15 largest alignments and visually inspected them for problems. Additionally, alignments for gene clusters from the highest divergence were visually inspected. All problematic alignments were discarded, and the involved data clusters were sub-clustered using BLASTCLUST with a higher similarity threshold. The similarity threshold was increased gradually with a step of $5 \%$ until a non-trivial sub-clustering was achieved. Sequences in new sub-clusters were re-aligned using procedures described above. These new alignments of sub-clusters were used in subsequent analyses.

\section{Species phylogeny}

To investigate the phylogenetic relationships of the sampled strains of streptococci, we concatenated alignments from the 504 data sets which were created by using the $30 \%$ similarity threshold and which contained exactly one gene sequence from each of the 12 genomes. Such homologous clusters represent putative single-copy genes. The resultant 556,899 nucleotide-long alignment was used to reconstruct a NJ tree using fast BIONJ algorithm
[55]. The branch lengths were estimated using the oneratio codon model (M0) from PAML [11].

\section{Maximum likelihood (ML) analyses}

The codeml program from the PAML [11] package was used for ML analyses based on Markov models of codon evolution. Branch lengths of the inferred ML trees, measured by number of expected nucleotide changes per codon, were estimated by using the simplest model M0 and then fixed in all further ML analyses to shorten the computational time. Model M0 assumes constant selective pressure across codon sites and over time. The selective pressure at the protein level was measured by $\omega$, the ratio of nonsynonymous to synonymous rates $d_{\mathrm{N}} / d_{S^{\prime}}$, with $\omega<1,=1$, or $>1$ indicating conserved, neutral or adaptive evolution respectively [e.g., [56]]. Models M1a, M2a, M7, and M8 of variable selective pressure across codon sites were used to estimate selective pressure and test for positive selection [57-59]. These models differ by the statistical distribution assumed for the $\omega$ ratio. For each homologous cluster, we performed two likelihood ratio tests (LRTs) for positive selection, comparing models that allow sites with $\omega>1$ (alternatives M2a and M8) with simpler models that do not (null models M1a and M7). Model M1a (nearly-neutral) assumes two site classes in proportions $p_{0}$ and $p_{1}=1-p_{0}$ : one with $\omega_{0}$ ratio estimated between 0 and 1 , and the other with $\omega_{1}$ fixed at 1 . Alternative model M2a (positive selection) extends the null model M1a by adding a proportion $p_{2}$ of positively selected sites with $\omega_{2}>1$, estimated from data. In the second LRT the alternative model M8 (beta $\& \omega$ ) extends the null model M7 (beta) by adding to the beta distribution for $\omega$ (defined on the interval $[0,1]$ ), an extra class of sites under positive selection with $\omega>1$. To ensure convergence to the best likelihood, all analyses were performed three times. The analyses took 3-4 weeks on linux cluster comprised of $10 \times 2.4 \mathrm{GHz}$ AMD Opteron processors.

The minimum number of sequences for informative analysis depends on the divergence levels. Based on computer simulations [17], reasonable statistical power can be achieved with as few as $4-5$ genomes for cases having divergences similar to those observed among Streptococcus strains.

Note that comparison of the absolute rate of synonymous $\left(d_{\mathrm{S}}\right)$ or nonsynoymous $\left(d_{\mathrm{N}}\right)$ substitutions among genes could be impacted by the way codon frequencies are handled [69]. However, it was not the purpose of this study to make quantitative comparisons among the genes; rather we sought simply to identify genes that had a history of positive diversifying selection. In this context, the interpretation of the $d_{\mathrm{N}} / d_{\mathrm{S}}$ ratio should not have been affected [70]. In the case of LGT, extreme values for nucleotide content reflect differences in the equilibrium state of the 
donor and recipient genomes involved in the LGT event. The biggest factor for such genes will be a very large estimate of the amount of codon evolution among those lineages. As noted in Results and Discussion, a large amount of codon evolution is compatible with both the hypothesis of LGT and strong positive Darwinian selection.

\section{Authors' contributions}

MA, JPB and ZY conceived the study. All authors participated in the design of the study. MA and KAD wrote the code that automated the genome-scale analyses, and carried out those analyses. All authors contributed to the statistical analysis and interpretation of results. MA, JPB and KAD were involved in drafting the manuscript. All authors have read and approved the final manuscript.

\section{Additional material}

\section{Additional file 1}

Gene clusters encoding proteins identified as having evolved under positive Darwinian selection pressure. Results of LRTs and additional information on gene clusters where positive selection was detected.

Click here for file

[http://www.biomedcentral.com/content/supplementary/14712148-7-154-S1.doc]

\section{Acknowledgements}

We thank Hong Gu for helpful discussions and Peter Cordes for technical support. This work was supported by a Biotechnology and Biological Sciences Research Council grant and an award from GlaxoSmithKline to ZY, a grant from the Natural Sciences and Engineering Research Council of Canada (DG298349) and a grant from the Canadian Foundation for Innovation (NOFI0394) both awarded to JPB. MA was supported by a fellowship from BBSRC, and KAD was supported in part by fellowships from Genome Canada and the Natural Sciences and Engineering Research Council of Canada.

\section{References}

I. Gill SR, Fouts DE, Archer GL, Mongodin EF, Deboy RT, Ravel J, Paulsen IT, Kolonay JF, Brinkac L, Beanan M, Dodson RJ, Daugherty SC, Madupu R, Angiuoli SV, Durkin AS, Haft DH, Vamathevan J, Khouri H, Utterback T, Lee C, Dimitrov G, Jiang L, Qin H, Weidman J, Tran K, Kang K, Hance IR, Nelson KE, Fraser CM: Insights on evolution of virulence and resistance from the complete genome analysis of an early methicillin-resistant Staphylococcus aureus strain and a biofilm-producing methicillin-resistant Staphylococcus epidermidis strain. J Bacteriol 2005, [ 87:2426-2438.

2. Chen SL, Hung CS, Xu J, Reigstad CS, Magrini V, Sabo A, Blasiar D, Bieri T, Meyer RR, Ozersky P, Armstrong JR, Fulton RS, Latreille JP, Spieth J, Hooton TM, Mardis ER, Hultgren SJ, Gordon JI: Identification of genes subject to positive selection in uropathogenic strains of Escherichia coli: a comparative genomics approach. Proc Natl Acad Sci USA 2006, 103:5977-82.

3. Whittam TS, Bumbaugh AC: Inferences from whole-genome sequences of bacterial pathogens. Curr Opin Genet Dev 2002, | 2:7| $9-725$.

4. Lan R, Reeves RR: Intraspecies variation in bacterial genomes: the need for a species genome concept. Trends Microbiol 2000, 8:396-40I.
5. Ruoff K, Whiley RA, Beighton D: Streptococcus. In Manual of clinical microbiology Volume I. 8th edition. Edited by: Murray PR, Baron EJ, Jorgensen JH, Pfaller MA, Yolken RH. Washington, D.C: ASM Press; 2003:405-42I.

6. Orhieula CJ, Radin JN, Sublett JE, Gao G, Kaushal D, Tuomanen El: Microarray analysis of pneumococcal gene expression during invasive disease. Infect Immun 2004, 72:5582-5596.

7. Smoot JC, Barbian KD, Van Gompel JJ, Smoot LM, Chaussee MS, Sylva GL, Sturdevant DE, Ricklefs SM, Porcella SF, Parkins LD, Beres SB, Campbell DS, Smith TM, Zhang Q, Kapur V, Daly J, Veasy LG, Musser JM: Genome sequence and comparative microarray analysis of serotype MI 8 group A Streptococcus strains associated with acute rheumatic fever outbreaks. Proc Natl Acad Sci USA 2002, 99:4668-73.

8. Glaser P, Rusniok C, Buchrieser C, Chevalier R, Frangeul L, Msadek T, Zouine M, couve E, Lalioui L, Poyart C, Trieu-Cuot P, Kunst F: Genome sequence of Streptococcus agalactiae, a pathogen causing invasive neonatal disease. Mol Microbiol 2002, 45: $1499-513$.

9. Klein DL: Pneumococcal disease and the role of conjugate vaccines. Microb Drug Resist 1999, 8:275-282.

10. Guindon S, Gascuel O: A simple, fast, and accurate algorithm to estimate large phylogenies by maximum likelihood. Syst Biol 2003, 52:696-704.

II. Yang Z: PAML: a program package for phylogenetic analysis by maximum likelihood. Comput Appl Biosci 1997, 3:555-556.

12. Garcia-Vallvé $S$, Romeu A, Palau J: Horizontal gene transfer in bacterial and archaeal complete genomes. Genome Res 2000, I0:1719-1725.

13. Xie G, Bonner CA, Bretin T, Gottardo R, Keyhani NO, Jensen RA: Lateral gene transfer and ancient paralogy of operons containing redundant copies of tryptophan-pathway genes in Xylella species and in heterocystous cyanobacteria. Genome Biol 2003, 4:RI4.

14. Täpp J, Thollesson M, Herrmann B: Phylogenetic relationships and genotyping of the genus Streptococcus by sequence determination of the RNase P RNA gene, rnpB. Internat J of Syst Evol Microbiol 2003, 53:186I-I87I.

15. Marri PR, Hao W, Golding GB: Gene gain and gene loss in Streptococcus: Is it driven by habitat? Mol Biol Evol 2006, 23:2379-2391.

16. Delorme C, Poyart C, Ehrlich SD, Renault P: Extent of horizontal gene transfer in evolution of streptococci of the salivarius group. J Bacteriol 2007, 189:|330-134|.

17. Anisimova M, Bielawski JP, Yang Z: Accuracy and power of the likelihood ratio test in detecting adaptive molecular evolution. Mol Biol Evol 200I, I 8: I585-I592.

18. Anisimova M, Bielawski JP, Yang Z: Accuracy and power of Bayes prediction of amino acid sites under positive selection. Mol Biol Evol 200I, 19:950-958.

19. Anisimova M, Nielsen R, Yang Z: Effect of recombination on the accuracy of the likelihood method for detecting positive selection at amino acid sites. Genetics 2003, 164:1229-1236.

20. Shriner D, Nickle DC, Jensen MA, Mullins Jl: Potential impact of recombination on sitewise approaches for detecting positive natural selection. Genet Res 2003, 8I: I I5-2I.

21. Wilson DJ, McVean G: Estimating diversifying selection and functional constraint in the presence of recombination. Genetics 2006, 172: |41।-1425.

22. Martin DP, Williamson C, Posada D: RDP2: recombination detection and analysis from sequence alignments. Bioinformatics 2005, $21: 260-262$.

23. Thanassi JA, Hartman-Neumann SL, Dougherty TJ, Dougherty BA, Pucci MJ: Identification of I I 3 conserved essential genes using a high-throughput gene disruption system in Streptococcus pneumoniae. Nucleic Acid Res 2002, 30:3 152-3162.

24. Federle MJ, Mclver KS, Scott JR: A response regulator that represses transcription of several virulence operons in the group A Streptococcus. J Bacteriol 1999, 18 1:3649-3657.

25. Graham MR, Smoot LM, Lux Migliaccio CA, Virtaneva K, Sturdevant DE, Porcella SF, Federle MJ, Adams GJ, Scott JR, Musser JM: Virulence control in group A Streptococcus by a two-component gene regulatory system: Global expression profiling and in vivo infection modeling. Proc Natl Acad Sci USA 2002, 99:13855-13860. 
26. Rasmussen M, Müller H-P, Björck L: Protein GRAB of Streptococcus pyogenes regulates proteolysis at the bacterial surface by binding $\alpha_{2}$-macroglobulin. J Biol Chem 1999, 274:I5336-I5344.

27. Toppel AW, Rasmussen M, Rohde M, Medina E, Chhatwal GS: Contribution of protein G-related $\alpha_{2}$-macroglobulin-binding protein to bacterial virulence in a mouse skin model of group $A$ streptococcal infection. J Infect Dis 2003, 187:1694-1703.

28. Collin M, Svensson MD, Sjöholm AG, Jensenius JC, Sjöbring U, Olsén $A$ : EndoS and SpeB from Streptococcus pyogenes inhibit immunoglobulin-mediated opsonophagocytosis. Infect Immun 2002, 70:6646-665I.

29. Collin M, Olsén A: EndoS, a novel secreted protein from Streptococcus pyogenes with endoglycosidase activity on human IgG. EMBO 200I, 20:3046-3055

30. Collin M, Olsén A: Effect of SpeB and EndoS from Streptococcus pyogenes on human immunoglobulins. Infect Immun 200I, 69:7187-7189.

31. Åkesson P, Moritz L, Truedsson M, Christensson B, von Pawel-Rammingen $U$ : IdeS, a highly specific immunoglobulin G (IgG)cleaving enzyme from Streptococcus pyogenes, is inhibited by specific IgG antibodies generated during infection. Infect Immun 2006, 74:497-503.

32. Kwon H-Y, Kim S-W, Choi M-H, Ogunniyi AD, Paton JC, Park S-H, Pyo S-N, Rhee D-K: Effect of heat shock and mutations in clpL and clpP on virulence gene expression in Streptococcus pneumoniae. Infect Immun 2003, 7 I:3757-3765.

33. Kwon H-Y, Ogunniyi AD, Choi M-H, Pyo S-N, Rhee D-K, Paton JC: The ClpP protease of Streptococcus pneumoniae modulates virulence gene expression and protects against fatal pneumococcal challenge. Infect Immun 2004, 72:5646-5653.

34. Ibrahim YM, Kerr AR, Silva NA, Mitchell TJ: Contribution of the ATP-dependent protease ClpCP to the autolysis and virulence of Streptococcus pneumoniae. Infect Immun 2005, 73:730-740.

35. Gottesman S: Regulation by proteolysis: developmental switches. Curr Opin Microbiol 1999, 2: I42-I 47.

36. Wickner S, Maurizi MR, Gottesman S: Posttranslational quality control: folding, refolding, and degrading proteins. Science 1999, 286: I888-1893.

37. Charpentier E, Novak R, Tuomanen E: Regulation of growth inhibition at high temperature, autolysis, transformation and adherence in Streptococcus pneumoniae by clpC. Mol Microbiol 2000, 37:717-726.

38. Polissi A, Pontiggia A, Fager G, Altieri M, Mottl H, Ferrari L, Simon D: Large-scale identification of virulence genes from Streptococcus pneumoniae. Infect Immun 1998, 66:5620-5629.

39. Sandler SJ, Marians KJ: Role of PriA in replication fork reactivation in Escherichia coli. J Bacteriol 2000, 182:9-13.

40. McGlynn P, Lloyd RG: Rescue of stalled replication forks by RecG: Simultaneous translocation on the leading and lagging strand templates supports an active DNA unwinding model of fork reversal and Holliday junction formation. Proc Natl Acad Sci USA 200I, 98:8227-9234.

41. Buchmeier NA, Libby SJ, Xu Y, Loewen PC, Switala J, Guiney DG, Fang FC: DNA repair is more important than catalase for Salmonella virulence in mice. / Clin Invest 1995, 95: 1047-1053.

42. Savarnapanya AE, Stein MA: DNA base excision repair potentiates the protective effect of Salmonella Pathogenicity island 2 within macrophages. Microbiol 2005, I 5 I:557-567.

43. Seigneur M, Bidnenko V, Ehrlich SD, Michel B: RuvAB acts at arrested replication forks. Cell 1998, 95:419-430.

44. Kang J, Tavakoli D, Tschumi A, Aras RA, Blaser MJ: Effect of host species on recG phenotypes in Helicobacter pylori and Escherichia coli. J Bacteriol 2004, 1 86:7704-77|3.

45. Jiang S-M, Cieslewicz MJ, Kasper DL, Wessels MR: Regulation of virulence by a two-component system in group B Streptococcus. | Bacteriol 2005, I87: I |05-IIII3.

46. Mascher T, Zähner D, Merai M, Balmelle N, de Saizieu AB, Hakenbeck R: The Streptococcus pneumoniae cia regulon: CiaR target sites and transcription profile analysis. I Bacteriol 2003, 185:60-70

47. Voyich JM, Sturdevant DE, Braughton KR, Kobayashi SD, Lei B, Virtaneva K, Sorward DW, Musser JM, DeLeo FR: Genome-wide protective response used by group A Streptococcus to evade destruction by human polymorphonuclear leukocytes. Proc Natl Acad Sci USA 2003, 100:1996-200I.
48. Woolhouse ME, Webster JP, Domingo E, Charlesworth B, Levin BR: Biological and biomedical implications of the co-evolution of pathogens and their hosts. Nat Genet 2002, 32:569-577.

49. Aris-Brosou S: Determinants of adaptive evolution at the molecular level: the extended complexity hypothesis. Mol Biol Evol 2005, 22:200-209.

50. Marra A, Asundi J, Bartilson M, Lawson S, Fang F, Christine J, Wiesner C, Brigham D, Schneider WP, Hromockyj AE: Differential fluorescence induction analysis of Streptococcus pneumoniae identifies genes involved in pathogenesis. Infect Immun 2002, 70:1422-1433.

51. Hava DL, Camilli A: Large-scale identification of serotype 4 Streptococcus pneumoniae virulence factors. Mol Microbiol 2002, 45: 1390-I 405.

52. Altschul SF, Gish W, Miller W, Myers EW, Lipman DJ: Basic local alignment search tool. J Mol Biol 1990, 215:403-4I0.

53. Notredame C, Higgins D, Heringa J: T-Coffee: A novel method for multiple sequence alignments. J Mol Biol 2000, 340:385-395.

54. Swofford DL: PAUP* Phylogenetic analysis using parsimony (*and other methods) Version 4 Sunderland, MA: Sinauer Associates; 200I.

55. Gascuel O: BIONJ: an improved version of the NJ algorithm based on a simple model of sequence data. Mol Biol Evol 1997 , | 4:685-695.

56. Yang Z, Bielawski JP: Statistical methods for detection molecular adaptation. Trends Ecol Evol 2000, I5:496-503.

57. Nielsen R, Yang Z: Likelihood models for detecting positive selected amino acid sites and application to the HIV-I. Genetics 1998, 148:929-936.

58. Yang Z, Nielsen HR, Goldman N, Pedersen AMK: Codon-substitution models for heterogeneous selection pressure at amino acid sites. Genetics 2000, I 55:43I-449.

59. Yang Z, Wong WSW, Nielsen R: Bayes empirical bayes inference of amino acid sites under positive selection. Mol Biol Evol 2005, 22:1107-III8.

60. Ferretti JJ, McShan WM, Ajdic D, Savic DJ, Savic G, Lyon K, Primeaux C, Sezate S, Suvorov AN, Kenton S, Lai HS, Lin SP, Qian Y, Jia HG, Najar FZ, Ren Q, Ahu H, Song L, White J, Yuan Z, Clifton SW, Roe $B A$, McLaughlin R: Complete genome sequence of an MI strain of Streptococcus pyogenes. Proc Natl Acad Sci USA 200I, 98:4658-4663.

6I. Beres SB, Sylva GL, Barbian KD, Lei B, Hoff JS, Mammarella ND, Liu MY, Smoot JC, Porcella SF, Parkins LD, Campbell DS, Smith TM, McCormick JK, Leung DY, Schlievert PM, Musser JM: Genome sequence of a serotype M3 strain of group A Streptococcus: phage-encoded toxins, the high-virulence phenotype, and clone emergence. Proc Natl Acad Sci USA 2002, 99:10078-83.

62. Nakagawa I, Kurokawa K, Yamashita A, Nakata M, Tomiyasu Y, Okahashi N, Kawabata S, Yamazaki K, Shiba T, Yasunaga T, Hayashi H, Hattori M, Hamada S: Genome sequence of an M3 strain of Streptococcus pyogenes reveals a large-scale genomic rearrangement in invasive strains and new insights into phage evolution. Genome Res 2003, 13:1042-55.

63. Banks DJ, Porcella SF, Barbian KD, Beres SB, Philips LE, Voyich JM, DeLeo FR, Martin JM, Somerville GA, Musser JM: Progress toward characterization of the group A Streptococcus metagenome: complete genome sequence of a macrolide-resistant serotype M6 strain. J Infect Dis 2004, 190:727-738.

64. Tettelin H, Masignani V, Cieslewicz MJ, Eisen JA, Peterson S, Wessels MR, Paulsen IT, Nelson KE, Margarit I, Read TD, Madoff LC, Wolf AM, Beanan MJ, Brinkac LM, Daugherty SC, DeBoy RT, Durkin AS, Kolonay JF, Madupu R, Lewis MR, Radune D, Fedorova NB, Scanlan D, Khouri H, Mulligan S, Carty HA, Cline RT, Van Aken SE, Gill J, Scarselli M, Mora M, lacobini ET, Brettoni C, Galli G, Mariani M, Vegni F, Maione D, Rinaudo D, Rappuoli R, Telford JL, Kasper DL, Grandi G, Fraser CM: Complete genome sequence and comparative genomic analysis of an emerging human pathogen, serotype V Streptococcus agalactiae. Proc Natl Acad Sci USA 2002, 99:| $239 \mid-6$

65. Hoskins J, Alborn WE Jr, Arnold J, Blaszczak LC, Burgett S, DeHoff BS, Estrem ST, Fritz L, Fu DJ, Fuller W, Geringer C, Gilmour R, Glass JS, Lhoja H, Kraft AR, Lagace RE, LeBlanc DJ, Lee LN, Lefkowitz EJ, Lu J, Matsushima P, McAhren SM, McHenney M, McLeaster K, Mundy CW, Nicas TI, Norris FH, O'Gara M, Peery RB, Robertsons GT, Rockey P, Sun PM, Winkler ME, Yang Y, Young-Bellido M, Zhao G, Zook CA, Baltz RH, Jaskunas SR, Rosteck PR Jr, Skatrud PL, Glass Jl: 
Genome of the bacterium Streptococcus pneumoniae strain R6. J Bacteriol 200I, 183:5709-57I7.

66. Tettelin H, Nelson KE, Paulsen IT, Eisen JS, Read TD, Peterson S, Heidelberg J, DeBoy RT, Haft DH, Dodson RJ, Durkin AS, Gwinn M, Kolonay JF, Nelson WC, Peterson JD, Umayam LA, White O, Salzberg SL, Lewis MR, Radune D, Holtzapple E, Khouri H, Wolf AM, Utterback TR, Hansen CL, McDonald LA, Feldblyum TV, Angiuoli S, Dickinson T, Hickey EK, Hold IE, Loftus BJ, Yang F, Smith HO, Venter JC, Dougherty BA, Morrison DA, Hollingshead SK, Fraser CM: Complete genome sequence of a virulent isolate of Streptococcus pneumoniae. Science 200I, 293:498-506.

67. Ajdic D, McShan WM, McLaughlin RE, Savic G, Chang J, Carson MB, jPrimeaux C, Tian R, Kenton S, Jia H, Lin S, Qian Y, Li S, Zhu H, Najar F, Lai H, White J, Roe BA, Ferretti JJ: Genome sequence of Streptococcus mutan UAI59, a carcinogenic dental pathogen. Proc Natl Acad Sci USA 2002, 99:14434-9.

68. Bolotin A, Quinquis B, Renault P, Sorokin A, Ehrlich SD, Kulakauskas S, Lapidus A, Goltsman E, Mazur M, Pusch GD, Fonstein M, Overbeek R, Kyprides N, Purnelle B, Prozzi D, Ngui K, Masuy D, Hancy F, Burteau S, Boutry M, Delcour J, Goffeau A, Hols P: Complete sequence and comparative genome analysis of the dairy bacterium Streptococcus thermophilus. Nat Biotechnol 2004, 22: $1523-1524$

69. Aris-Brosou S, Bielawski JP: Large-scale analyses of synonymous substitution rates can be sensitive to assumptions about the process of mutation. Gene 2006, 378:58-64.

70. Yang Z: Computational Molecular Evolution. Oxford University Press; 2006:284

Publish with Bio Med Central and every scientist can read your work free of charge

"BioMed Central will be the most significant development for disseminating the results of biomedical research in our lifetime. "

Sir Paul Nurse, Cancer Research UK

Your research papers will be:

- available free of charge to the entire biomedical community

- peer reviewed and published immediately upon acceptance

- cited in PubMed and archived on PubMed Central

- yours - you keep the copyright

Submit your manuscript here:

http://www.biomedcentral.com/info/publishing_adv.asp
BioMedcentral 